\title{
Self-Organized Discotic Liquid Crystals for High-Efficiency Organic Photovoltaics
}

\author{
L. Schmidt-Mende, ${ }^{1}$ A. Fechtenkötter, ${ }^{2}$ K. Müllen, ${ }^{2}$ E. Moons, ${ }^{3}$ \\ R. H. Friend, ${ }^{1}$ J. D. MacKenzie ${ }^{1}$
}

Self-organization of liquid crystalline and crystalline-conjugated materials has been used to create, directly from solution, thin films with structures optimized for use in photodiodes. The discotic liquid crystal hexa-perihexabenzocoronene was used in combination with a perylene dye to produce thin films with vertically segregated perylene and hexabenzocoronene, with large interfacial surface area. When incorporated into diode structures, these films show photovoltaic response with external quantum efficiencies of more than 34 percent near 490 nanometers. These efficiencies result from efficient photoinduced charge transfer between the hexabenzocoronene and perylene, as well as from effective transport of charges through vertically segregated perylene and hexabenzocoronene $\pi$ systems. This development demonstrates that complex structures can be engineered from novel materials by means of simple solution-processing steps and may enable inexpensive, high-performance, thin-film photovoltaic technology.

Solution-processible conjugated organic materials combine the electronic properties of semiconductors and the processibility of polymeric materials. This makes them suitable for commercial applications in optoelectronic technologies, in which the adaptability, simplicity, and low cost of manufacture make them extremely attractive. In general, crystalline molecular organic materials exhibit better transport properties than do their polymeric counterparts. At the time of this writing, the highest field-effect charge carrier mobilities reported for organic materials at room temperature were achieved in single-crystal perylene transistors (1). In addition, impressive photovoltaic efficiencies have been achieved in evaporated organic double-layer and doped molecular devices (2-5). Howev$\mathrm{er}$, in the field of photovoltaics, in which low manufacturing cost is essential, organics that can be solution-processed at low temperatures have a unique advantage. Here we have sought to realize the high performance of three-dimensionally ordered crystalline organics in a solution-processed photovoltaic device by exploiting the unique combination of facile one-dimensional (1D) transport and mesoscopic ordering of a discotic liquid crystal phase separated in a blend with a soluble, crystal network-forming organic. This work has demonstrated that discotic liquid crystalline (LC) organics can be used for interesting and useful optoelectronic applications, in

${ }^{1}$ Cavendish Laboratory, University of Cambridge, Madingley Road, Cambridge CB3 OHE, UK. ${ }^{2}$ MaxPlanck-Institut für Polymerforschung Ackermannweg 10, D-55128 Mainz, Germany. ${ }^{3}$ Cambridge Display Technology, Greenwich House, Madingley Road, Cambridge $\mathrm{CB} 3 \mathrm{OHJ}$, UK. which high performance is attained through the exploitation of intermolecular and mesoscopic ordering.

High-efficiency organic photovoltaic devices derive their performance from two basic elements: (i) the dissociation within the active semiconductor layers of the photogenerated electronic excited states (termed excitons), and (ii) the transport of the resulting separated charge to the electrodes. It is generally found that it is necessary to use a heterojunction formed between electron- and hole-accepting semiconductors in order to ionize these strongly bound excitons (6). Blends of soluble conjugated polymers and blends of polymers and polymer-C60 molecules that exhibit photoinduced charge separation and that phase-separate into interpenetrating networks have been used to create devices with external quantum efficiencies (EQEs) of 5 to $10 \%(7,8)$. Recent work describes polymer/methanofullerene blend devices with maximum EQEs up to $50 \%(9)$. The high degree of photoluminescence (PL) quenching generally observed in these systems suggests that transport, not exciton dissociation, limits performance. By using lamination to create a multilayer device with an idealized charge transport structure from spin-coated films that are soluble in a common solvent, EQEs as high as $29 \%$ were demonstrated (10). However, yield and scaling difficulties may limit the commercial application of a lamination technique. Impressive efficiencies have also been demonstrated with small-molecule devices relying on either carefully controlled layer deposition of charge transfer pairs $(2-4)$ or the use of extrinsic dopant-mediated charge transfer in a high-mobility molecular matrix (5). However, both of these require vacuum processing of the organic layers.

We report here the solution processing of small-molecule materials, which we use to engineer self-organized photovoltaic structures with maximum EQEs of $>34 \%$ and power efficiencies of up to $\sim 2 \%$. Microscopy, spectral characteristics, and basic modeling indicate that a nearly ideal, vertically segregated structure, with a large interfacial area between the electron-accepting perylene and hole-accepting hexabenzocoronene components, has been achieved. In this way, multicomponent self-organization of novel discotic LC materials, inducing a high interfacial surface area morphology and a vertically ordered composition, has been applied to organic photodiodes in such a way that complex ideally ordered structures can be realized with a potentially manufacturable technique. We emphasize that this one-step process makes use of self-organization in an organic liquid crystal and crystal network to achieve structures that would otherwise require multiple evaporation, lamination, or spinning steps to create stratified organic structures, and also produces a larger interfacial area between donor and acceptor material.

The materials used in this study include soluble perylene $N, N^{\prime}$-bis(1-ethylpropyl)-3,4,9,10perylenebis (dicarboximide) (perylene) and a discotic, LC, hexaphenyl-substituted hexabenzocoronene $\left(\mathrm{HBC}-\mathrm{PhC}_{12}\right)(11)$. Perylene diimides are known to possess high electron mobilities $(12,13)$ and have been used previously as an electron acceptor and transport material in small molecules and dye-polymer blend devices $(6,14)$. Electron diffraction has been used to show the high crystallinity of the perylene in spin-coated films. The perylene dye and HBC$\mathrm{PhC}_{12}$ are both soluble in chloroform. HBC$\mathrm{PhC}_{12}$ is discotic $\mathrm{LC}$ at room temperature. In this LC phase, columns of disc-shaped molecules are formed, which can allow quasi-1D transport of charge carriers and excitons along the columns with high carrier mobility. Mobilities as high as $\Sigma \mu_{1 \mathrm{D}}=0.22 \mathrm{~cm}^{2} \mathrm{~V}^{-1} \mathrm{~s}^{-1}$ $\left(\Sigma \mu_{1 \mathrm{D}}\right.$, the mobility resulting from all) have been measured for intracolumnar charge carrier motion in $\mathrm{HBC}-\mathrm{PhC}_{12}$ (15). Figure 1A shows the chemical structure of $\mathrm{HBC}-\mathrm{PhC}_{12}$, along with a simplified diagram of the $\pi-\pi$ stacking configuration. The LC ordering in spin-coated HBC- $\mathrm{PhC}_{12}$ thin films is supported by the observation of micron-scale domain structures with polarized light microscopy. Cross-polarized light microscopy in blend films is made difficult by the polarizing effects of the perylene crystallites. The same LC phase for $\mathrm{HBC}-\mathrm{PhC}_{12}$ is expected in the blend films, in which the distinctly crystalline perylene component is separated into micron-scale crystallites. Figure 1B shows the structure of the perylene molecule and an electron diffraction image taken from a 


\section{R E P O R T S}

film spin-coated from xylene. The densest ring of diffraction spots from perylene crystallites corresponds to a spacing of approximately 3.5 $\AA$, which is similar to the $\pi-\pi$ stacking direction spacing observed in many conjugated molecules (16). Cyclic voltammetry was performed on thin films of these materials to determine the highest occupied molecular orbital (HOMO) level. The lowest unoccupied molecular orbital (LUMO) level is then calculated from the optical gap obtained from the absorption spectrum. These measurements indicate a HOMO level of $5.25 \mathrm{eV}$ and a LUMO level of $2.64 \mathrm{eV}$ for $\mathrm{HBC}-\mathrm{PhC}_{12}$, which has a relatively large band gap of $2.61 \mathrm{eV}$ (Fig. 1C). The perylene HOMO and LUMO energies were similarly determined as 5.32 and $3.29 \mathrm{eV}$, respectively. From HBC$\mathrm{PhC}_{12}$ to perylene, which has a high electron affinity as a result of the electron-withdrawing diimide bridges, there is $0.65 \mathrm{eV}$ LUMO step and $0.07 \mathrm{eV}$ HOMO step. These band-edge offsets promote exciton dissociation and charge transfer (17), which was verified by PL quenching. The PL of the perylene is quenched by a factor of 11.6 when pumped at a wavelength of $488 \mathrm{~nm}$ with the addition of $\mathrm{HBC}-\mathrm{PhC}_{12}$ to make a $1: 1$ blend by weight. The absorption of $\mathrm{HBC}-\mathrm{PhC}_{12}$ at this wavelength is considered negligible.

Both the hexabenzocoronene and the perylene exhibit characteristic thin-film morphologies upon spin coating from solutions of $15 \mathrm{~g} /$ liter. Figure $2 \mathrm{~A}$ is a tapping-mode atomic force microscopy (AFM) image of a pure $\mathrm{HBC}-\mathrm{PhC}_{12}$ surface on 5- and $20-\mu \mathrm{m}$ (inset) scales; it shows a smooth textured surface with a root mean square (rms) surface roughness of $<10 \mathrm{~nm}$. Polarized light microscopy indicates the presence of large domains with typical in-plane length scales of hundreds of microns. In contrast, the pure perylene surface is considerably rougher, with characteristic micron-scale elongated crystallites as shown in Fig. 2B. The rms surface roughness of a typical perylene film was $>40 \mathrm{~nm}$, with some crystals protruding hundreds of nanometers from the surface. Spin coating of blend solutions of close to equal parts HBC$\mathrm{PhC}_{12}$ and perylene resulted in a markedly different surface morphology, indicating the presence of nearly continuous surface coverage of shortened perylene crystallites.

This can be seen in the AFM micrograph in Fig. 3A. Cross sections of blend films spin-coated on a $\mathrm{Si}(100)$ wafer were prepared by cleaving after immersion in liquid $\mathrm{N}_{2}$. The films were then imaged in a LEOS field-emission scanning electron microscope (SEM) capable of high-resolution, low-accelerating-voltage operation. Figure $3 \mathrm{~B}$ shows an electron micrograph of a $40 \%$ $\mathrm{HBC}-\mathrm{PhC}_{12}$ blend sample, imaged with the cleaved surface tilted $15^{\circ}$ degrees from normal to the incident electron beam $(1 \mathrm{kV})$.

B

Fig. 1. (A) The chemical structure of $\mathrm{HBC}-\mathrm{PhC}_{12}$, along with a simplified diagram of the $\pi-\pi$ stacking configuration. The intercolumnar distance $L=$ $34 \AA$ and the cofacial distance $d_{\mathrm{c}}=3.5 \AA$. (B) The structure of the perylene molecule and an electron diffraction image taken from a spin-coated xylene film. The densest ring of diffraction spots from perylene diimide crystallites corresponds to a spacing of approximately $3.5 \AA$ A. (C) HOMO and LUMO levels for HBC$\mathrm{PhC}_{12}$ and perylene. There is a $0.65-\mathrm{eV}$ LUMO offset
Stratification is clearly visible in the cross section, with a characteristic polycrystalline perylene sheet extending down the top surface of the film. Between the crystalline top surface and the substrate, a less-featured intermediate layer, possibly HBC$\mathrm{PhC}_{12}$, can be seen at the cleaved edge. Perylene crystallites with a cross section resembling that of the long crystallites observed by AFM in the pure perylene film (Fig. 2B) can be seen dispersed through the intermediate layer in the SEM image. Blends with more than a $20 \%$ imbalance in binary composition produced more complex morphologies, which suggested that continuous layers were stable only within composition or thickness limits.

The vertical segregation probably re-
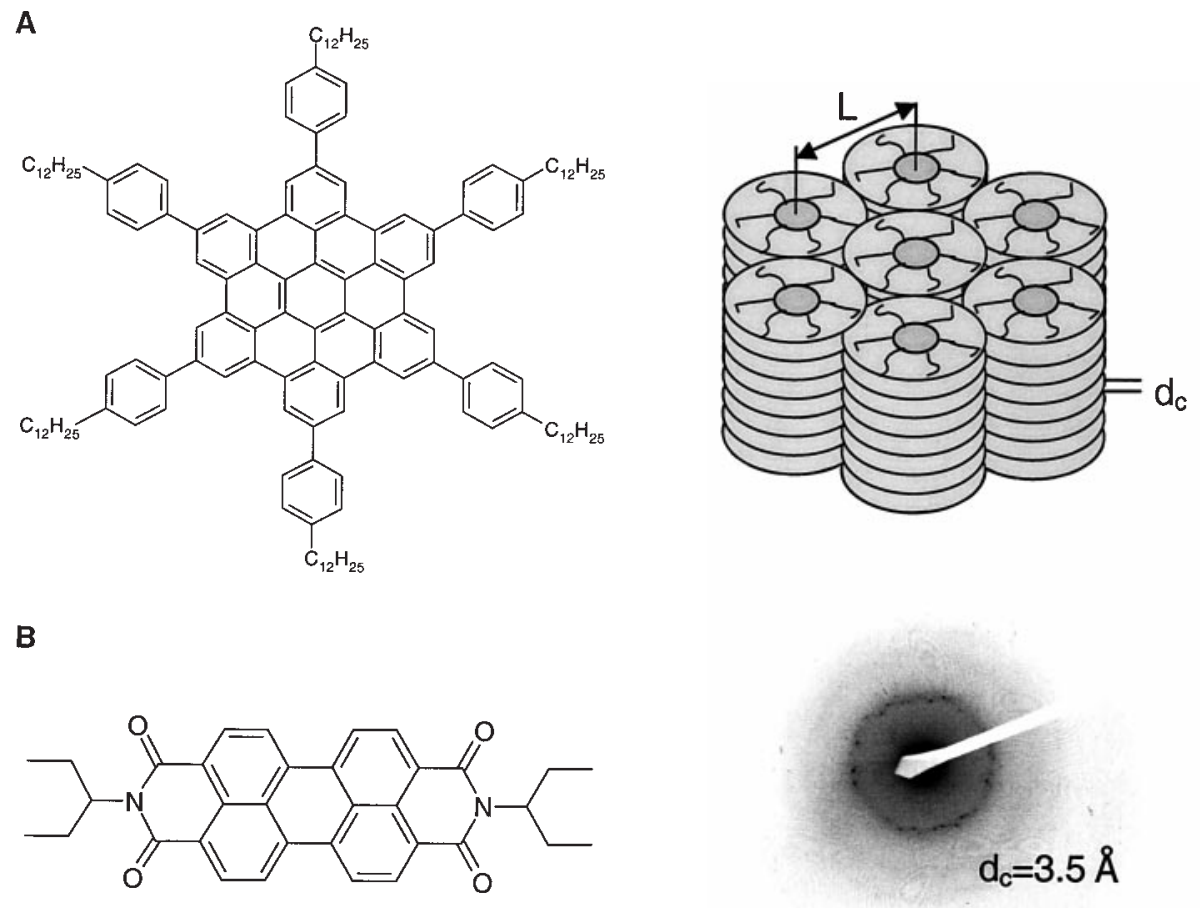
C sults from the complex interplay between differing solubilities and surface energies during the nonequilibrium spin-coating and solvent evaporation processes. Direct measurement of these quantities was difficult because of the limited amount of HBC$\mathrm{PhC}_{12}$ available and, in the case of the surface properties, the high surface roughness of the thin films. By inspection or through a simple quantitative group contribution approach following Hansen and others (18), substantial differences in the surface energies and the solubility in chloroform for the perylene and $\mathrm{HBC}-\mathrm{PhC}_{12}$ can be predicted. We consider that, because of the higher solubility of $\mathrm{HBC}-\mathrm{PhC}_{12}$, the perylene begins to crystallize near the free surface from which solvent is lost, whereas

and a $0.07-\mathrm{eV}$ HOMO offset between the two materials. 


\section{R E P O R T S}

HBC-PhC ${ }_{12}$ remains in solution. This could drive the formation of a solid perylene crystallite layer, which is less dense than the chloroform solution, "floating" at the interface between the drying film and the ambient, where the solvent concentration drops most rapidly. This layer may also act as a nucleation layer for subsequent crystallization as the solidification of the film proceeds. Demonstrations of vacuum epitaxy of perylene on hexa-peri-benzocoronene (19) are in accord with our results here that indicate the stability of a perylene/HBC- $\mathrm{PhC}_{12}$ multilayer structure in which the intermolecular process of photoinduced charge transfer can take place. Photodiodes formed by the evaporation of
$\mathrm{Al}$ onto $\mathrm{HBC}-\mathrm{PhC}_{12}$ :perylene blend films spin-coated from chloroform solutions (15 g/liter) onto $\mathrm{O}_{2}$ plasma-treated indium tin oxide (ITO) showed high photovoltaic quantum efficiencies. Figure 4A shows the EQE action spectra for a 40:60 HBC$\mathrm{PhC}_{12}$ :perylene device, along with the fraction of light that is absorbed in HBC$\mathrm{PhC}_{12}$, in perylene, and in a blend film. The EQE action spectrum peaks above 34\% near absorption bands associated with both materials, demonstrating that efficient charge transfer takes place from either component. Based on the differences between the EQE action spectrum, the fraction of light absorbed in the film of the individual components, and the blend de- vice as a function of wavelength, as shown in Fig. 4A, it can be inferred that the EQE for light absorbed in the perylene is somewhat higher than that in $\mathrm{HBC}-\mathrm{PhC}_{12}$. This may be due to a higher effective diffusion length for excitons in perylene or to other factors, such as impurity trapping. Further work is required to elucidate this issue. Double-layer devices made by vacuum deposition of the perylene layer on top of a solution-deposited HBC-PhC 12 layer show qualitatively the same spectral behavior. However, the absolute efficiencies were substantially lower, by one order of magnitude. This is thought to be due to the smaller interfacial area at the evaporated perylene/HBC-PhC ${ }_{12}$ boundary and
A

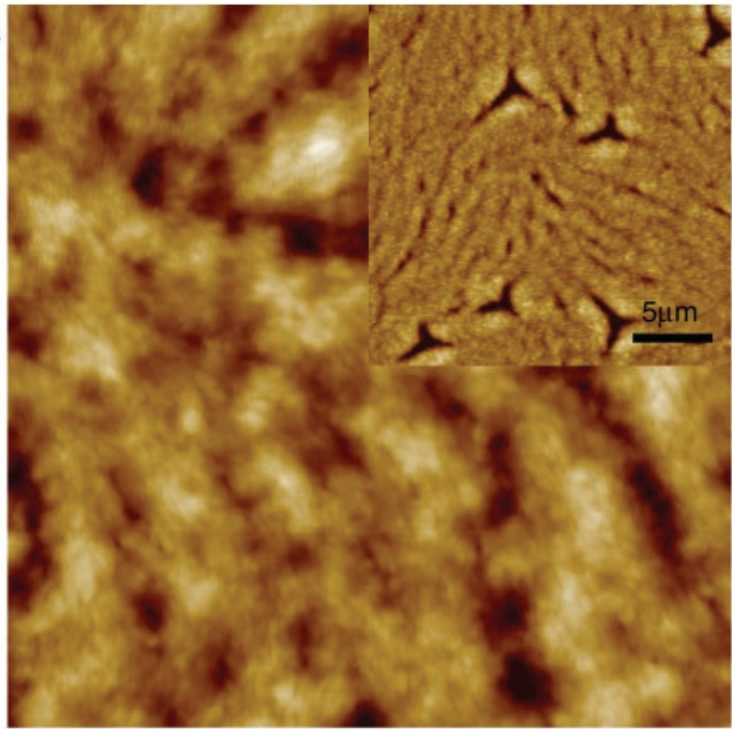

$1 \mu \mathrm{m}$

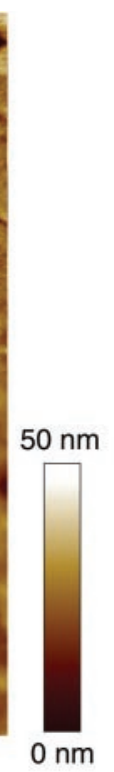

$0 \mathrm{~nm}$

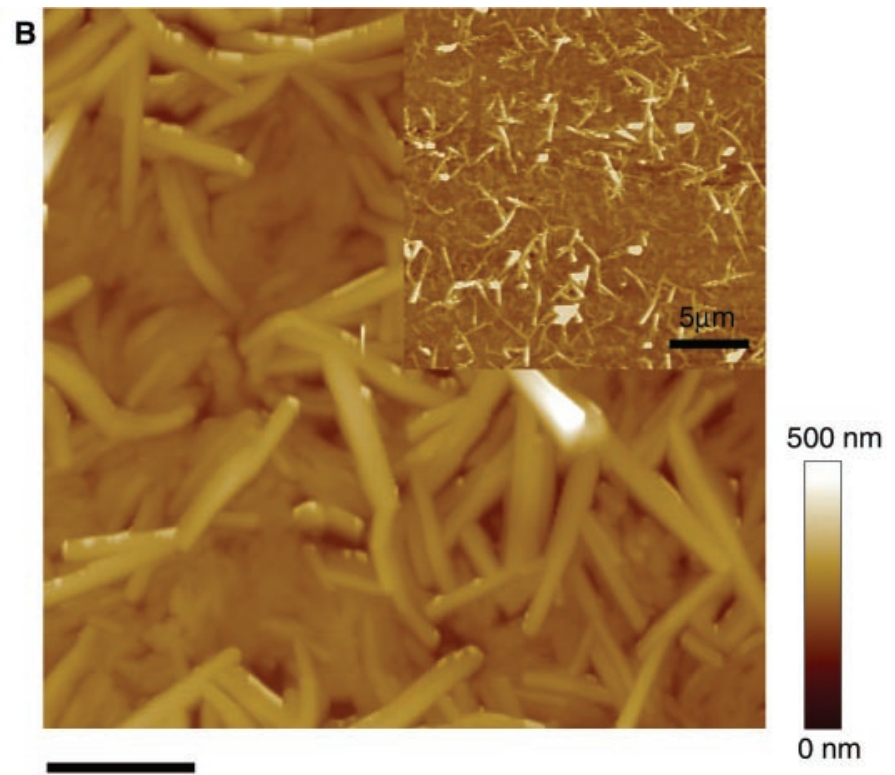

$1 \mu \mathrm{m}$

Fig. 2. Tapping-mode AFM image of (A) a pure $\mathrm{HBC}-\mathrm{PhC}_{12}$ surface and (B) a pure perylene diimide surface on 5 - $\mu \mathrm{m}$ and 20 - $\mu \mathrm{m}$ (insets) scales.

A

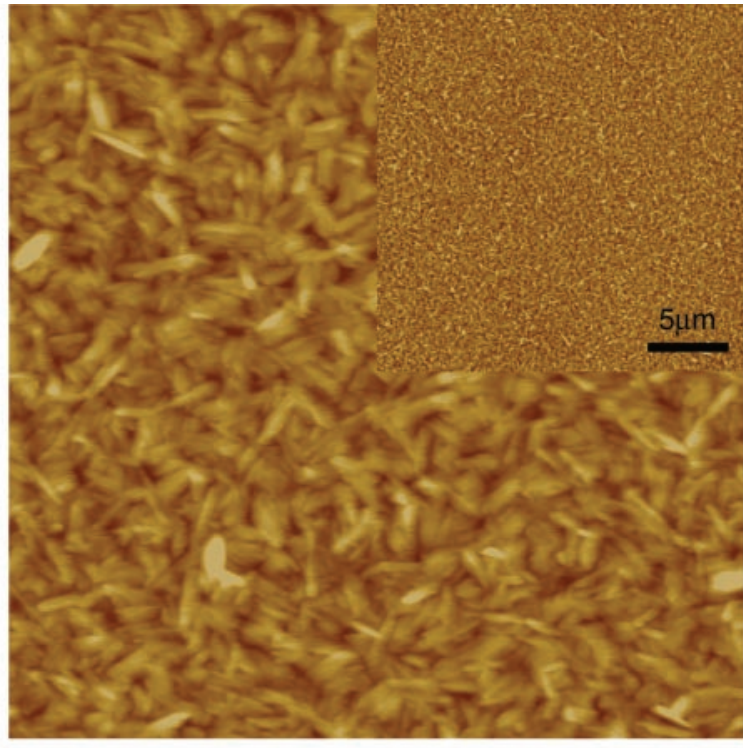

$1 \mu \mathrm{m}$
B

$20 \mathrm{~nm}$

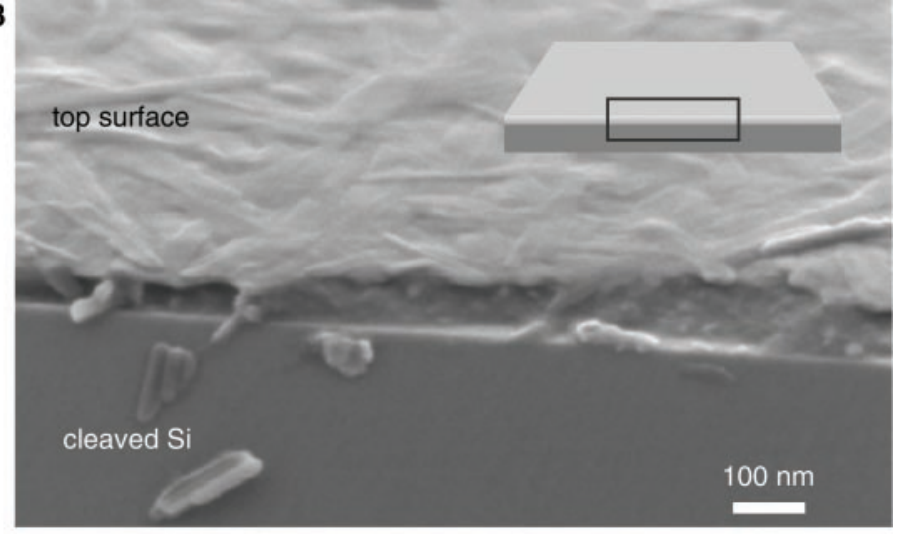

Fig. 3. (A) Tapping-mode AFM image of a film spin-coated from a 40:60 blend solution of $\mathrm{HBC}-\mathrm{PhC}_{12}$ and perylene diimide. (B) Field-emission SEM image of a $40 \% \mathrm{HBC}-\mathrm{PhC}_{12}$ blend sample, imaged with the cleaved surface tilted $15^{\circ}$ from normal to the incident electron beam ( $\left.1 \mathrm{kV}\right)$. 


\section{REPOR T S}

provides further evidence for a large interfacial area in the devices made by the single solution step here. Figure 4B depicts the current-voltage characteristics for a 40:60 blend photodiode in the dark and under $490 \mathrm{~nm}$ illumination at $0.47 \mathrm{~mW} /$ $\mathrm{cm}^{2}$. The short circuit current and open circuit voltage under illumination were $-33.5 \mu \mathrm{A} / \mathrm{cm}^{2}$ and $0.69 \mathrm{~V}$, respectively, and the fill factor was $40 \%$. The power efficiency maximum was $1.95 \%$ at $490 \mathrm{~nm}$. Because of the observation of some saturation of the current density at light intensities above $1 \mathrm{~mW} / \mathrm{cm}^{2}$, and the imperfect match between the $\mathrm{HBC}-\mathrm{PhC}_{12}$ :perylene absorption spectrum and the solar spectrum, the EQE and power efficiency under solar conditions are expected to be lower. It is believed that the sublinearity at higher intensities is due to extrinsic degradation processes.

It is thought that the high EQEs measured for these solution-processed devices are partly due to the large interfacial area within the bilayer structure, strongly suggested by the microscopic investigations presented above, and possibly to the high exciton diffusion ranges in the separated $\mathrm{HBC}-\mathrm{PhC}_{12}$ and perylene regions. The high degree of PL quenching in the blend films
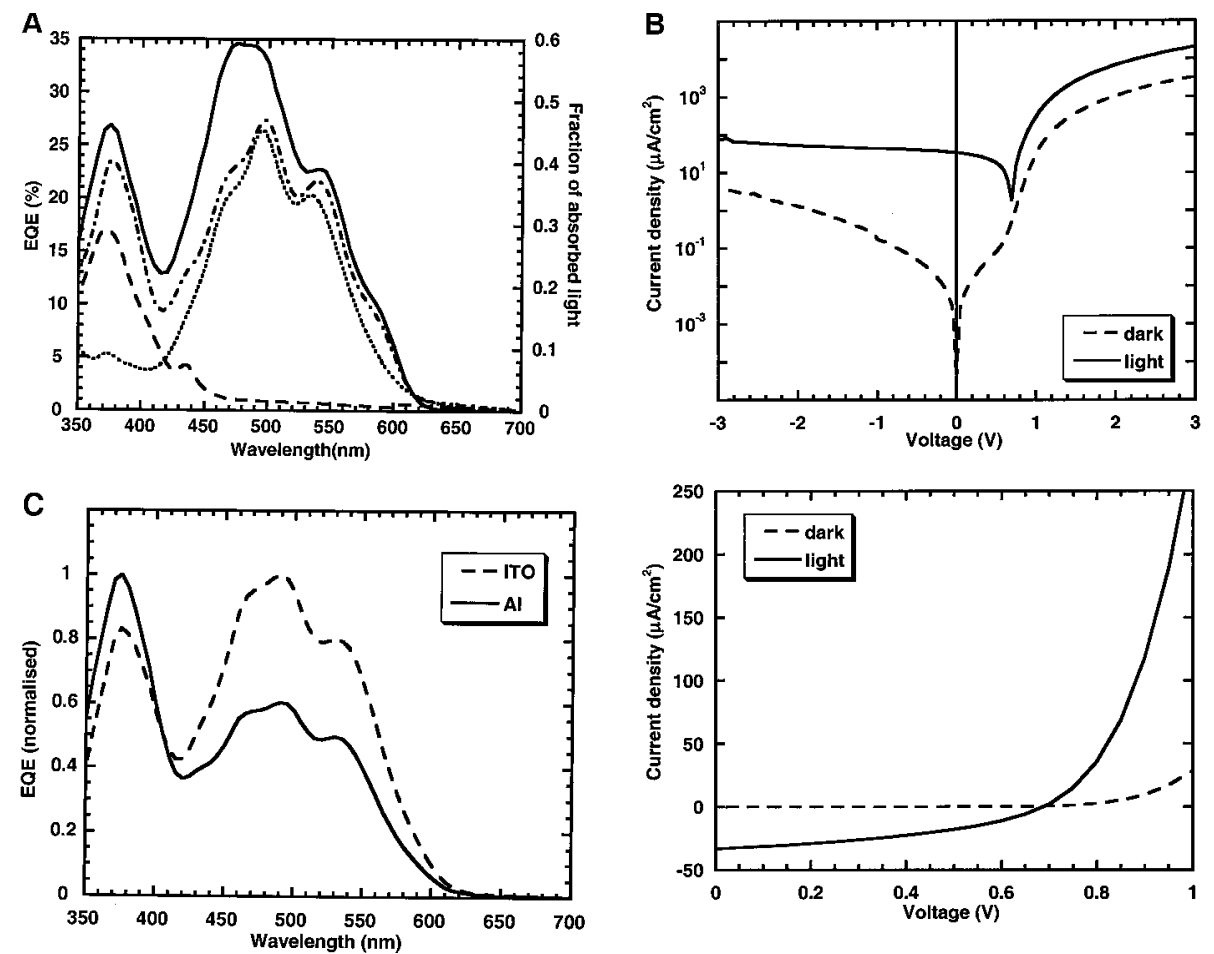

Fig. 4. (A) EQE action spectra for a 40:60 HBC-PhC 12 :perylene diimide blend (solid line) and the fraction of absorbed light in an $\mathrm{HBC}-\mathrm{PhC}_{12}$ film (dashed line), a perylene film (dotted line), and a 40:60 blend film (dash-dotted line). (B) Current-voltage characteristics for this device in the dark and under illumination at $490 \mathrm{~nm}$. The short circuit current and open circuit voltage under illumination are $-33.5 \mu \mathrm{A} / \mathrm{cm}^{2}$ and $0.69 \mathrm{~V}$, respectively, and the fill factor is $40 \%$. The diode has a rectification ratio of $8.3 \times 10^{2}$ at $2 \mathrm{~V}$. (C) Normalized EQE action spectra taken under illumination through the ITO and Al electrodes, respectively, corrected for the absorption of the contacts and glass. tion can take place. The observed effect is not the result of filtering or absorption effects due to a larger effective optical path length in the case of illumination through the ITO. In these cases, the opposite trend in the action spectrum peaks would be expected upon changing the path length, and this was confirmed in measurements on thicker devices. Qualitatively the same spectral behavior was also observed in twolayer devices made by vacuum deposition of the perylene layer on top of a solutionprocessed $\mathrm{HBC}-\mathrm{PhC}_{12}$ layer.

The use of solution-processible discotic LC material in combination with a crystal network-forming material leads to stable solid-film structures with useful morphologies for optoelectronic applications. The self-organization can be exploited in organic photovoltaics to produce optimized structures for photoexciton dissociation and charge transport in a solution-processed materials system with this combination of crystal- and liquid crystal-forming materials. This development brings together the simplicity of single-step solution processing with an efficient combination of molecular materials that can show unique ordering on intermolecular, mesoscopic, and interphase scales and can spontaneously develop a multilayer transport structure. Further optimization could prove this route to be a way toward a commercially viable organic photovoltaic system.

References and Notes

1. J. H. Schön, C. Kloc, B. Batlogg, Appl. Phys. Lett. 77, 23 (2000).

2. C. W. Tang, Appl. Phys. Lett. 48, 2 (1986).

3. J. J. M. Halls, K. Pichler, R. H. Friend, S. C. Moratti, A. B. Holmes, Appl. Phys. Lett. 68, 22 (1996).

4. L. Chen et al., Adv. Mater. 12, 18 (2000).

5. J. H. Schön, C. Kloc, E. Bucher, B. Batlogg, Nature 403 , 6768 (2000).

6. J. J. M. Halls et al., Phys. Rev. B 60, 5721 (1999).

7. J. J. M. Halls et al., Nature 376, 6540 (1995).

8. G. Yu, J. Gao, J. C. Hummelen, F. Wudl, A. J. Heeger, Science 270, 5243 (1995).

9. S. E. Shaheen et al., Appl. Phys. Lett. 78, 6 (2001).

10. M. Granström et al., Nature 395, 6699 (1998).

11. A. Fechtenkötter, K. Saalwächter, M. A. Harbison, K. Müllen, H. W. Spiess, Angew. Chem. Int. Ed. 38, 20 (1999).

12. J. H. Schön, C. Kloc, B. Batlogg, Appl. Phys. Lett. 77, 23 (2000).

13. W. C. Struijk et al., J. Am. Chem. Soc. 122, 45 (2000).

14. J. J. Dittmer et al., Sol. Energy Mater. Sol. Cells 61, 53 (2000).

15. A. M. van de Craats et al., Adv. Mater. 11, 17 (1999).

16. J. Mizuguchi, J. Appl. Phys. 84, 8 (1998).

17. V. Cleave al., Adv. Mater. 13, 1 (2001).

18. J. E. Mark, Physical Properties of Polymers Handbook (AIP Press, Woodbury, NY, 1996), chap. 16.

19. T. Schmitz-Hübsch, Surf. Sci. 445, 358 (2000).

20. Partial funding was provided by Sony International (Europe) and the European Commission under BriteEuram contract BRPR-CT97-046. The authors thank A. Yasuda, G. Nelles, and T. Miteva (Sony International) for fruitful discussions and A. C. Arias, J. J. M. Halls, and C. Ramsdale (Cavendish Laboratory, Univ. of Cambridge) for their help. 